

\title{
PERSPECTIVAS EN TORNO A LA FORMACIÓN DOCENTE Y LA POSIBILIDAD DE UNA CAPACITACIÓN Y ACTUALIZACIÓN CONSTANTE: UNA MIRADA DESDE LOS ACTORES EN UNA UNIVERSIDAD MEXICANA.
}

\author{
Perspectives around teaching training and the possibility of a training and constant update: a \\ look at the actors at a mexican university.
}

Perspectivas sobre a formação de professores e a possibilidade de formação e atualização constante: uma visão dos atores de uma universidade mexicana.

RECIBIDO:25 MAYO 2017

es

\section{RESUMEN}

La formación docente ha sido muy cuestionada a consecuencia de los resultados académicos que reflejan las evaluaciones nacionales e internacionales en los diferentes niveles de la educación básica y media superior. La capacidad del maestro ha sido considerada la variable sustancial donde se concentra la responsabilidad de la realidad actual que vive el proceso educativo. El propósito del presente artículo es realizar un análisis del proceso de formación docente, valorar la eficacia de los mecanismos de formación, capacitación y actualización, analizando su pertinencia, su atención a los requerimientos de la sociedad actual, asî como al modelo filosófico de maestro que se pretende formar. El estudio consistió en la aplicación de un cuestionario a docentes de instituciones educativas del nivel básico y medio superior; se realizó un análisis estadístico con apoyo del programa (SPSS), la selección de la muestra fue casuística, considerando como criterio docentes que se encontraran en servicio. Los mecanismos de capacitación y actualización docente han partido, hasta el momento, de la ausencia de un diagnóstico de necesidades; así también, aunque la carrera se haya elevado a nivel de licenciatura y los estudios a nivel posgrados en educación hayan proliferado, los resultados son insuficientes; de la misma manera se considera que la implicación y el compromiso del maestro son factores esenciales para una enseñanza eficaz.

PALABRAS CLAVE: formación docente, profesionalización, actitud, innovación.
EVALUADO:17JULIO 2017,

22 AGOTO 2017,14 ENERO 2019

Santiago Delgado Coronado

Doctor en Educación. Universidad Continente Americano Plantel Abasolo.

sdc_1966@hotmail.com

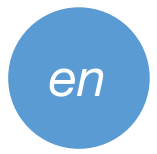

\section{ABSTRACT}

Teacher training has been questioned as a result of academic results that reflect national and international assessments at different levels of the average basic and higher education. The ability of the teacher has been considered the substantial variable to the responsibility of the current reality of the educational process focuses. The purpose of this article is to analyze the process of teacher training, evaluate the effectiveness of the mechanisms of formation, training and updating analyzing their relevance, their attention to the requirements of today's society as well as the philosophical model teacher who It aims to train. The study consisted of a questionnaire to teachers in educational institutions of basic and higher average; a statistical analysis support program (SPSS) was performed, the selection of the sample was casuistry, considering as criteria, teachers were in service. Mechanisms teacher training and updating departed, so far, in the absence of a needs assessment; well, although the race has risen at undergraduate and postgraduate level studies in education have proliferated, the results are insufficient; in the same way, it is considered that the involvement and commitment of the teacher are essential for effective teaching.

KEYWORDS: Teacher training, professionalization, attitude, innovation.
ACEPTADO:30 ENERO 2019

\section{RESUMO}

A formação de professores tem sido muito questionada como resultado dos resultados académicos reflectidos nas avaliações nacionais e internacionais nos diferentes níveis do ensino básico e secundário. A capacidade do professor tem sido considerada a variável substancial onde se concentra a responsabilidade pela realidade atual do processo educativo. $\mathrm{O}$ objetivo deste artigo é realizar uma análise do processo de formação de professores, para avaliar a eficácia dos mecanismos de formação, formação e atualização, analisando a sua pertinência, sua atenção às exigências da sociedade atual, bem como o modelo filosófico do professor que se pretende formar. $\mathrm{O}$ estudo consistiu na aplicação de um questionário para professores de instituições de ensino dos níveis básico e secundário superior; uma análise estatística foi realizada com o apoio do programa (SPSS); a seleção da amostra foi casuística, considerando como critérios, professores que estavam em serviço. Os mecanismos de formação e atualização de professores têm se baseado, até agora, na ausência de uma avaliação das necessidades; da mesma forma, embora as carreiras tenham aumentado até o nível de graduação e os estudos de pós- graduação em educação tenham proliferado, os resultados são insuficientes; da mesma forma, o envolvimento e o compromisso dos professores são considerados fatores essenciais para um ensino eficaz.

PALAVRAS CHAVE: formação de professores, profissionalização, atitude, inovação.

PARA CITAR ESTE ARTÍCULO/TO CITE THIS ARTICLE/PARA CITAR ESTE ARTIGO:

Delgado-Coronado, S. (2019). PERSPECTIVAS EN TORNO A LA FORMACIÓN DOCENTE Y LA POSIBILIDAD DE UNA CAPACITACIÓN Y ACTUALIZACIÓN 


\section{INTRODUCCIÓN}

El sistema educativo nacional se encuentra inmerso en una crisis de resultados académicos, las evaluaciones con estándares nacionales e internacionales muestra esta gran debilidad de los procesos de formación en los diferentes contextos. En función del análisis en los diferentes ámbitos y niveles, se concibe a la formación docente como el eje principal de las deficiencias proyectadas por estas pruebas aplicadas a estudiantes mexicanos.

La formación se concibe como un proceso social y cultural que obedece al carácter de la integridad del desarrollo de la capacidad transformadora humana que se da en la dinámica de las relaciones entre los sujetos en la sociedad, en constante y sistemática relación, capaz de potenciar y transformar su comportamiento en el saber, hacer, ser y convivir (Vargas, 2010, p.3); la capacitación se define como el proceso de enseñanza-aprendizaje donde se adquieren conocimientos y se desarrollan destrezas; por último, la actualización se considera como la actividad de ponerse al día, estar a la vanguardia, acceder a la nueva información en el campo de la educación.

Desde una perspectiva holística, se establece que son muchos los factores que intervienen en el desarrollo del proceso educativo; el sistema se torna muy complejo, confluyen factores metodológicos, psicológicos, sociales, curriculares, culturales, etc. De la misma manera, se considera importante la participación y el compromiso de los actores de este proceso: alumnos, maestros, padres de familia, autoridades, y sociedad en general, ya que cada uno de ellos participa de manera directa o indirecta en la formación de los miembros de la sociedad.

En México se han propuesto varías prácticas de profesionalización, capacitación y actualización docente, algunas que conllevan vacíos teóricos, académicos y con poca relación práctica hacia una formación para la vida; la última propuesta se orienta al desarrollo de competencias, con el propósito de que la escuela ofrezca un servicio de calidad, ya que esta implica profesores bien preparados y con una actualización constante.

\section{PERCEPCIÓN SOBRE LA FORMACIÓN DOCENTE}

"Varios estudios señalan al factor docente como elemento clave de la transformación educativa, actor principal de la renovación de los modelos de enseñanza" (Aguerrondo, 2004; Fullan, 2002; Vaillant 2005). Cualquiera sea el cambio que se impulse, el enfoque en el cual se oriente, entendidos o no por parte de la sociedad, "los docentes siempre son interpelados desde algún lugar, ya sea que se les ubique como ejecutores, mediadores de lo planificado por los expertos o en calidad de protagonistas activos y reflexivos de la transformación" (Vezub, 2007, p.3).

La formación del maestro ha sido muy cuestionada, se señala la carencia de un sinnúmero de competencias que el docente del siglo XXI debe poseer; para la OCED y la UNESCO, el 'docente deseado', el 'docente eficaz' es caracterizado como un sujeto polivalente, profesional competente, agente de cambio, practicante reflexivo, profesor investigador, intelectual critico e intelectual transformador (Barth, 1990; Delors et al., 1996; Hargreaves, 1994; Gimeno, 1992; Jung, 1994; OCDE, 1991; Schon, 1992; UNESCO, 1990, 1998): que domine los saberes y contenidos que pretende enseñar, sabe facilitar el aprendizaje, sabe e interpreta el currículo escolar, comprende la cultura de cada contexto, desarrolla una pedagogía activa, trabaja en equipo, etc.; con base en los resultados proyectados por las evaluaciones con estándares nacionales e internacionales, se puede considerar que los procesos de formación no han logrado alcanzar ese ideal de maestro eficaz deseado.

Anderson señala: "un docente efectivo es aquel que con bastante consistencia logra objetivos enfocados sobre el aprendizaje de sus estudiantes, sea de forma directa o indirecta" (1991, p.18); desde esta perspectiva, el maestro debe lograr un aprendizaje de calidad, ya que este es el propósito general de la educación. Para esto es necesario que el docente desarrolle un conjunto de competencias que le permitan conllevar el desarrollo de su práctica docente bajo los principios de calidad; Dunkin (1997), define la competencia docente como el conocimiento y las habilidades necesarias, y el desempeño docente como la forma en que este se conduce durante el proceso de enseñar.

Algunos estudios realizados muestran que en las escuelas públicas los docentes poseen una educación muy deficitaria, algunos tienen habilidades básicas limitadas, reciben escasa formación y perciben bajas remuneraciones (Arregui et al., 1996; Vaillant, 2004b). Lo anterior debería orientar la realización de un análisis del proceso
Perspectivas en torno a la formación docente y la posibilidad de una capacitación y actualización constante: una mirada desde los actores en una universidad mexicana.

| Panorama | pp.34-41 | Volumen 13 | Número 24 | Enero-Junio | 2019 
de formación docente a las instituciones encargadas de dicha preparación, a los planes y programas de instrucción, a los mecanismos de capacitación y actualización del profesorado.

Santiago

Delgado

Coronado

El segundo Informe del PREAL concluía que: En promedio, los maestros latinoamericanos no se preparan adecuadamente para su función. Tienen menos horas de formación profesional que sus contrapartes de los países desarrollados y la capacitación que reciben suele ser de mala calidad. Estas afirmaciones contrastan con la creciente importancia dada por los gobiernos de la región al tema docente. A pesar de los esfuerzos y de las inversiones realizadas en la materia, los resultados distan de ser los esperados. Ya es común hablar del "bajo impacto" que tiene la capacitación de los docentes en la transformación y mejoramiento de las prácticas de enseñanza (Vezub, 2007, p. 5).

Investigaciones han demostrado que los docentes con una actitud más favorable hacia las innovaciones, al desarrollo profesional, a la formación permanente, a la autoevaluación como una estrategia de reflexión y mejora, además de su capacidad para integrar nuevas ideas en su propia práctica, así como también su ausentismo, la rotación laboral, entre otros, son aspectos que influyen de forma importante en el éxito de los estudiantes y sus actitudes hacia la escuela (Harris, 1998; Stronge, 2002; Thijs \& Verkuyterm, 2009).

\section{LA FORMACIÓN DEL MAESTRO MEXICANO}

Si consideramos partir de la época precolombina, la educación de los hijos se encontraba a cargo de los padres de familia, donde el propósito de dicha formación consistía en educar el corazón, es decir, atender los sentimientos, las emociones del ser humano; por otra parte, orientada a instruir sobre los deberes principales que un joven debiese aprender desde la perspectiva de género, y sobretodo, educar la sensibilidad humana en los valores del respeto, la obediencia, etc.

Durante la época de la colonia, la educación fue eminentemente religiosa, el propósito central era formar a los niños y jóvenes bajo los principios de la doctrina cristiana, atendiendo a los principios de obediencia, el amor al prójimo y, de la misma manera, al conocimiento de la lectura, escritura y operaciones básicas de cálculo; dicho proceso estaba a cargo de religiosos, por lo que hasta este momento no aparecía como oficio la docencia.

El establecimiento de la Compañía Lancasteriana, en 1822, significó una oportunidad para la sociedad mexicana que buscaba disminuir los índices de analfabetismo. La responsabilidad de formar a los educandos durante la operatividad de esta compañía estaba a cargo de docentes contratados por el ayuntamiento, bajo ciertos indicadores que se establecieron con el fin de que el maestro pudiera impartir clase en esa época. Las primeras escuelas normales se establecieron bajo el régimen lancasteriano —refiriéndonos a normales como el lugar en el que se norma la enseñanza - en un curso que duraba de cuatro a seis meses.

Una vez considerado el magisterio como carrera, en 1887 se inauguró la Escuela Normal para Profesores en la Ciudad de México, que tuvo la responsabilidad de formar a los docentes de esa época. La formación del profesor de primaria, a partir de 1921, ha tenido dos momentos claves que influyeron en su preparación: uno es el maestro rural, misionero, comprometido con una concepción humanista y social de la profesión docente, ya que durante el periodo revolucionario el problema del analfabetismo era muy alto, se necesitaba personal para emprender campañas de alfabetización, por lo que se reclutó a todos aquellos que tan solo supieran leer y escribir para emprender esas jornadas de enseñanza; es cuando aparecen la misiones culturales, caravanas de personas que iban de pueblo en pueblo enseñando a leer y escribir e instruyendo en algunos oficios. Hasta este momento, se podría manifestar que los docentes no eran objeto de ninguna capacitación, mucho menos una actualización.

El otro momento es cuando se otorga a los estudios normalistas el nivel de licenciatura, cambiando su estatus con un gran número de asignaturas y el excesivo énfasis en las disciplinas teóricas y especulativas.

En 1958, Jaime Torres Bodet funda el Instituto Federal de Capacitación del Magisterio, cuyo propósito era mejorar las capacidades intelectuales de los maestros en ejercicio y acrecentar sus conocimientos de los contenidos educativos, es decir, la preocupación en esos momentos era que los docentes dominaran los conocimientos a impartir dentro de las aulas; pero aún en las décadas de los 50 y 60 se contrataban personas que solo 
hubieran terminado los estudios primarios o secundarios. Para 1958 existían 18 mil docentes que prestaban sus servicios en la federación, 9.000 solo tenían certificado de primaria, 3.000 tenían estudios incompletos de secundaria, 3.000 habían egresado de escuelas normales rurales y solo 2.000 de ellos habían sido formados en escuelas normales de plan completo.

La preparación y capacitación docente ha sufrido cambios de acuerdo con el proyecto educativo implementado en cada época, llámese nacionalista, socialista, plan de once años, tecnología educativa, modernización educativa, reforma integral, etc., tratando de unificar sus planes de estudio para la formación del maestro y favorecer el desarrollo de ciertas habilidades y conocimientos requeridos en esta etapa de la historia de la formación del magisterio. El ejemplo se observa con el surgimiento de la Universidad Pedagógica Nacional, con el plan 75: la malla curricular estaba orientada a fortalecer el dominio de conocimientos básicos de nivel superior.

Con el desarrollo del plan 99 se orientaba a favorecer, además del conocimiento, el proceso metodológico; las técnicas de enseñanza aparecen como los instrumentos y herramientas que ayudarían a conducir el proceso. La tecnología educativa da relevancia a la implementación de las técnicas y dinámicas de estudio, pero sin dar prioridad a la capacitación del maestro; en educación básica, fue hasta el proyecto de modernización educativa cuando quedó plasmada en los planes y programas de estudio la necesidad de que el maestro se capacitara de manera permanente para el desempeño de su trabajo docente.

El diseño de los mecanismos de capacitación y actualización docente quedó registrado bajo el nombre de Talleres generales de actualización, mismos que se impartían al inicio del ciclo escolar; se seleccionaban temas que se consideraban necesarios para fortalecer, lo que duró varios ciclos escolares, pero los resultados seguían siendo insuficientes. Posteriormente se pensó en la planeación estratégica como la panacea que resolvería el problema de los malos resultados; dentro de este contexto, se ha apostado por el diseño y puesta en práctica de la ruta de mejora. Al inicio del ciclo escolar se dedica una semana para su diseño, con la esperanza de que esto apoye a mejorar los resultados académicos, pero no se ha visto gran avance al respecto: jacaso se consolida la perspectiva de Lipman? "Las escuelas de pedagogía parecieran sufrir continuas crisis de identidad, parecen estar constantemente probándose una nueva personalidad según lo que esté más de moda en cada momento" (Lipman, M., Sharp A., \& Oscanyan, F. 1992, p.48).

\section{REQUERIMIENTOS DE LA FORMACIÓN DOCENTE}

Se comparte la necesidad e importancia de que el maestro reciba una formación adecuada a los requerimientos de la sociedad actual y una capacitación constante que fortalezca los conocimientos, las habilidades y destrezas para el desempeño de su labor docente, teniendo presente el tipo de alumno que se pretende formar; el docente debe apropiarse y tener presente el enfoque formativo para el desarrollo de su práctica, dominar el área de conocimiento que pretenda enseñar, debe demostrar un dominio eficaz de las técnicas y estrategias de enseñanza acorde con el modelo educativo que se intenta implantar, propiciar actitudes de laboriosidad, exigencia, hábito de trabajo intenso, etc.

Es entendible que la sociedad vaya evolucionando, buscando adaptarse a las nuevas exigencias de los adelantos científicos, tecnológicos, sociales, económicos, etc., pero es necesario generar modelos de formación docente apropiados al momento histórico que se viva, atendiendo a los requerimientos sociales de la época; si la educación del futuro ha de tener solidez, la educación de los profesores exigirá mayor integridad que la que tiene actualmente (Lipman, Sharp, \& Oscanyan, 1992, p.48).

Otro aspecto que se debe considerar es la formación de formadores. Para Gorodokin (2006, p.2), esta "debe procurar la formación de sujetos competentes". Así, la formación docente es más que una sumatoria de conocimientos adquiridos, ya que durante el proceso se estructuran representaciones, identificaciones, métodos y actitudes que impactan en el sujeto en formación tanto en el plano cognoscitivo como en el socio-afectivo.

Para la Secretaría de Educación Pública existen competencias básicas que el docente debe poseer para el desarrollo de su práctica pedagógica, entre ellas: dominio de los contenidos que pretende enseñar, creación de ambientes de aprendizaje, trabajo colaborativo, manejo e incorporación de las TIC, dominio de una segunda lengua, etc.
Perspectivas en torno a la formación docente y la posibilidad de una capacitación y actualización constante: una mirada desde los actores en una universidad mexicana
| Panorama | pp.34-41 | Volumen 13 | Número 24 | Enero-Junio | 2019 
Santiago

Delgado

Coronado

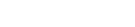

Vaillant (2004), señala que los formadores de las instituciones de formación docente no cuentan con una preparación especial para sus funciones, algunos no tienen experiencia de aula, porque ocuparon sus cargos apenas culminaron sus estudios en la misma institución; un gran porcentaje de ellos siguen enseñando tal como fueron formados: con dictados, exposiciones magistrales, usando pocos libros y brindando a los futuros docentes escasa experiencia práctica de métodos de enseñanza efectivos.

"El docente, desde el deber ser de su actuación profesional, debe reflexionar sobre su práctica pedagógica para mejorarla y/o fortalecerla y desde esa instancia elaborar nuevos conocimientos" (Díaz, 2006, p.89). Se deben plantear preguntas tales como ¿qué estamos haciendo?, ¿cómo lo estamos haciendo?, ¿qué tan bien o mal lo estamos haciendo?, ¿promovemos la investigación?, etc.

La hipótesis que se planteó para el presente estudio: si no existe claridad en el modelo docente que requiere la sociedad moderna, y este no es conocido ni entendido por el maestro, no se logrará una formación profesional eficaz ni pertinente.

El objetivo para lograr en la presente investigación: analizar el proceso de formación y capacitación docente para proponer alternativas de mejora en la generación de aprendizajes eficaces.

\section{METODOLOGÍA}

Se aplicó un cuestionario diseñado con preguntas de carácter cerrado. En dos de ellas se solicita que el entrevistado argumente o justifique sus respuestas; la finalidad de esto es obtener mayores elementos que permitan valorar cualitativamente la variable ‘¿A qué le atribuyes la buena o mala efectividad de los cursos tomados?', así como también a la variable ‘ ¿El sistema educativo tendrá bien claro el modelo de maestro que quiere formar?; la primera ofrece elementos que permiten identificar posibles causas sobre la poca o mucha efectividad de la herramienta de actualización o capacitación docente; la segunda muestra la perspectiva que tiene el maestro sobre la claridad del prototipo de formación docente que pueda tener o no el sistema educativo.
Se encuestaron 67 docentes adscritos a seis instituciones de nivel primaria, tres de educación preescolar y una de nivel medio superior; dos de estas pertenecen al sistema estatal y ocho al federal; del total, cinco se encuentran ubicadas en la zona rural y cinco en la zona urbana. Sus características son variadas, seis son de organización completa y las restantes son multigrados. Una de las principales características para considerar a los investigados fue que estuviesen en servicio y que pertenecieran al sistema de educación básica y media superior; la razón por la que se consideró este criterio fue que en el esquema de educación básica, en el año 2004 inició un proceso de transformación que pretendía reorientar el proceso educativo al desarrollo de competencias, por lo que el enfoque filosófico que sustenta estos cuatro niveles a los que se hace referencia es el mismo: desarrollo de competencias, sustentado en una filosofía humanista.

La elección de los investigados fue casuística; el criterio considerado para su selección fue ser maestro en servicio.

\section{ANÁLISIS DE RESULTADOS}

$\mathrm{El}$ instrumento que permitió recabar la información fue sometido al proceso de fiabilidad mediante la técnica del alfa de Cronbach, obteniendo una puntuación de 0.786

El mayor porcentaje de los investigados (42,1\%), se encuentran en un rango de entre uno y diez años de servicios, lo cual significa que son maestros jóvenes, recién egresados, con una formación más sólida con base en los enfoques que el propio sistema requiere. El 24,9\% se encuentra en el rango de entre once y veinte años de servicio; el 26,3\% entre veintiuno y treinta años. En cuanto al grado académico de los investigados, el $71,9 \%$ corresponde al grado de licenciatura, un $15 \%$ a la normal básica, y un $7 \%$ al posgrado; el porcentaje que corresponde al nivel licenciatura se asemeja a la sumatoria del porcentaje de docentes que cuentan hasta con veinte años de servicio, es decir, esta cantidad de maestros no han seguido estudiando, no se han preocupado por mejorar su preparación académica.

Otro de los datos que arrojó este estudio fue que el mayor porcentaje de docentes, $(35,1 \%)$, solo ha realizado entre uno y dos cursos en los últimos dos años; el 33,3\% menciona haber realizado cinco o más cursos. 
Lo curioso de este dato es que son los docentes de nivel medio superior los que expresan haberlos realizados, porque lo necesitan para la formación pedagógica y como requerimiento de la institución donde laboran. E1 29,8\% refiere haber participado entre tres y cuatro cursos durante los dos últimos años, considerando los talleres generales de actualización y los que se ofrecen de manera obligatoria; lo anterior muestra que es poca la actualización y capacitación que el docente recibe por la cantidad de cursos a los que asisten.

En cuanto a la variable 'valoración de la efectividad de los cursos o talleres', el 77,2\% los califica como buenos; el 50,9 \% atribuye esta valoración a la buena preparación del docente que lo imparte, mientras que el 26,3\% lo atribuye al interés de los participantes. Un $17,6 \%$ de maestros consideran los cursos o talleres como regulares y malos, atribuyéndolo a la falta de claridad en los contenidos y propósitos; mismas causas son señaladas por el 12,3 \% de docentes en cuanto al diseño del modelo filosófico sobre el tipo de maestro a formar.

La percepción que tienen los docentes sobre si los cursos recibidos promueven el desarrollo de las capacidades de reflexión, innovación, investigación, debate, así como si incluyen conocimientos, habilidades y actitudes que susciten el logro del perfil de egreso docente, el 57\% estableció que sí, el 8\% refirió que no y el 35\% expresó que solo en algunas veces. Lo anterior muestra que, en opinión de la mayoría de docentes encuestados, los cursos sí promueven las habilidades que se requieren desarrollar para aplicarlas dentro del aula, aunque algunos de ellos no logran identificar este tipo de habilidades, $y$ aún perciben los cursos muy verbalistas y desarrollados de manera tradicional.

Se cuestionó sobre si los cursos realizados por los docentes atendían sus necesidades de formación, pregunta a la que el 50,9\% expresó que sí, el 42,1\% dijo que solo algunas veces y el $7 \%$ señaló que no. Para un porcentaje de estos docentes, las temáticas ofrecidas no son interesantes.

La última pregunta que se les planteó fue: ¿el sistema educativo tendrá bien claro el modelo de maestro que pretende o quiere formar? $\mathrm{El} 36,8 \%$ dijo que no, el $35,1 \%$ expresó que solo algunas veces y el $28.1 \%$ señaló que sí.
Los entrevistados que respondieron negativamente justifican sus argumentos expresando que los cursos no consideran el contexto ni la realidad social en la que se desarrolla la práctica educativa, ya que este influye de manera determinante en ella; no se toma en cuenta la opinión de los docentes para el diseño de los planes y programas de estudio y, sobre todo, para la implementación de proyectos de reforma; no se tiene pleno conocimiento, por parte de quien diseña los proyectos educativos, de las verdaderas necesidades de formación que presentan los maestros, no existe congruencia entre lo que se dice y lo que se hace por parte de las autoridades educativas; hay quien considera que el modelo educativo que se desea implementar es muy limitado y copiado de otros contextos con características y particularidades muy distintas a las de México; otros expresan que constantemente se cambia de programas, y hace falta más actualización para conocer el sustento legal de las reformas existentes; algunos más manifiestan que son políticas sexenales y, por ende, ni el propio sistema tiene bien claro qué tipo de maestro desea formar.

Los docentes que ofrecen su respuesta afirmativa argumentan que las temáticas presentadas por los cursos fortalecen el tipo de docente a formar, porque van de acuerdo con las necesidades de aprendizaje que presenta el maestro; algunos dicen que se procura formar maestros con mayor capacidad para solucionar problemas.

\section{CONCLUSIONES}

Los resultados académicos son insuficientes, por lo que se comparte la necesidad de que el docente se capacite y actualice constantemente, pero este aspecto solo se consideró hasta el nacimiento del proyecto de modernización educativa, con la implementación de los talleres generales de actualización. Sin embargo, los resultados académicos no han mostrado un avance significativo, pues continúan las mismas prácticas dentro del aula; es decir, no ha habido eficacia en la consecución de los objetivos educativos.

Los mecanismos de capacitación y actualización docente parten de la ausencia de un diagnóstico serio de necesidades de formación. Solo hasta el año 2015 se implementaron las evaluaciones de diagnóstico para la permanencia en el sistema, pero aún no existen datos consolidados al respecto; existe una gran distancia entre
Perspectivas en torno a la formación docente y la posibilidad de una capacitación y actualización constante: una mirada desde los actores en una universidad mexicana. 
las intenciones formativas y las necesidades de la práctica educativa.

Santiago

Delgado

Coronado

La proliferación de los estudios de posgrado en el área de la educación se ha incrementado, la carrera de docente se elevó al grado de licenciatura, se han implementado talleres de actualización, etc., pero los resultados son insuficientes; los maestros expresan que, en cierta medida, estos cursos no atienden sus necesidades de preparación, carecen de un modelo de docente ejemplar; ¿cómo quiere el sistema educativo que sea el maestro?, ¿qué necesidades de formación tiene el maestro?, etc. Aunque la OCDE menciona una serie de indicadores que el docente debería atender, es preciso puntualizar sobre esto en cada curso, taller, diplomado o cualquiera que sea el mecanismo de capacitación con la finalidad de que el maestro siempre los tenga presente para que asimile, se apropie y los viva en cada proceso de su formación, capacitación o actualización.

Los planes y programas de estudio orientados a la formación de maestros han sido muy inconsistentes, los requisitos para la docencia se han dado únicamente desde el dominio de la lectura, la escritura y cálculos aritméticos, hasta el desarrollo de competencias para impartir la enseñanza; en la actualidad, los sistemas educativos solo atienden a los requerimientos establecidos por los organismos internacionales; Lipman (1992, p.48) señala, "las escuelas pedagógicas parecen sufrir continuas crisis de identidad, parecen estar constantemente probándose una nueva personalidad según lo que esté más de moda en cada momento". ¿Será que existe una crisis de identidad en la formación del maestro?

\section{Panorama REFERENCIAS}

3. Carro, A., Hernández, F., Lima, J., \& Corona, M. (2016). Formación profesional y competencias docentes en el estado de Tlaxcala. Revista Educación, 25(49), 7-28.
4. De Ibarrola M., Sañudo L., Moreno, M. \& Barrera, M. (2012). Los profesionales de la educación con formación de posgrado que México requiere. Informe, conclusiones y recomendaciones de los foros Internacionales de Formación de Investigadores y profesionales de Alto nivel en Educación. México: Departamento de Investigaciones educativas del CINVESTAV.

5. Díaz, V. (2006). Formación docente, práctica pedagógica y saber pedagógico. Laurus, 12(Ext), 88-103. Recuperado de: http://www.redalyc.org/ articulo.oa?id=76109906

6. Eurydice. (2011). La enseñanza de la lectura en Europa: contextos, politicas y prácticas. Agencia Ejecutiva en el Ámbito Educativo, Audiovisual y Cultural (EACE). España: Fareso.

7. Freire, P. (2010). Cartas para quien pretende enseñar. México: Editorial Siglo XXI.

8. Gorodokin, I. (2006). La formación docente y su relación con la epistemología. Revista Iberoamericana de Educación, 37(5), 1-10. Recuperado de: https://rieoei.org/RIE/article/ view/2691

9. Hunt, B. (2009). Efectividad del desempeño docente. Una reseña de la literatura internacional y su relevancia para mejorar la educación en América Latina. Santiago de Chile: Editorial San Marino. Recuperado de: http://www.radu.edu.ar/ Info/9\%20efectividad\%20doc.pdf

10. Marcelo, C. (2008). Evaluación de la calidad para programas completos de formación docente a través de estrategias de aprendizaje abierto y a distancia. RED, Revista de Educación a Distancia, 7(7), 1-6. Recuperado de: https://www.um.es/ ead/red/M7/marcelo.pdf

11. Murillo, F., Martínez, C., \& Hernández-Castilla R. (2001) Decálogo para una Enseñanza Eficaz. REICE, Revista Iberoamericana sobre Calidad, Eficacia y Cambio en Educación, 9(1), 6-27. Recuperado de: https://www.redalyc.org/articulo.oa?id=55118790002

12. Lipman, M., Sharp, A., \& Oscanyan, F. (1992). La práctica filosófica y la reforma educativa. Educar a los educadores en la filosofía en el aula. Madrid: Ediciones de la Torre.

13. Ornelas, C. (2000). El sistema educativo mexicano, la transición de fin de siglo. México: Fondo de cultura económica.

14. Pérez, M. (1999). ¿Qué necesidades de formación perciben los profesores? Tendencias pedagógicas, 4, 7-23. Recuperado de: https:// repositorio.uam.es/xmlui/handle/10486/4929

15 (2012). Plan y programas de estudio. Secretaría de Educación Pública México. 
16. Torres, M. (1999). Nuevo rol docente: ¿¿qué modelo de formación, para qué modelo educativo? En Aprender para el futuro: Nuevo marco de

17. Vargas, L. (2010). La formación docente. Congreso y la posibilidad de Iberoamericano de Educación, metas 2021.

Recuperado de: http://www.chubut.edu.ar/ descargas/secundaria/congreso/DOCENTES/ R0103_Magyoly.pdf

18. Vezub, L. (2007). La formación y el desarrollo profesional docente frente a los nuevos desafíos los actores en de la escolaridad. Profesorado. Revista de curríuna universidad . Recuperado de: http://www.ugr.es/local/recfpro/ rev111ART2.pdf 